\title{
Outcome in disappearing colorectal cancer liver metastases during oxaliplatin-based chemotherapy
}

\author{
TOMOJIRO ONO, HIDEYUKI ISHIDA, KENSUKE KUMAMOTO, NORIMICHI OKADA and KEIICHIRO ISHIBASHI
}

\author{
Department of Digestive Tract and General Surgery, Saitama Medical Center, \\ Saitama Medical University, Kawagoe, Saitama 350-8550, Japan
}

Received April 5, 2012; Accepted July 16, 2012

DOI: $10.3892 / \mathrm{ol} .2012 .842$

\begin{abstract}
Some colorectal cancer liver metastases (CLMs) disappear on serial imaging during chemotherapy and the optimal treatment strategy for such lesions remains undetermined. The purpose of this study was to investigate the outcome in disappearing CLMs, as few studies have focused on this topic, with conflicting results. Among 125 patients with CLMs treated with modified FOLFOX6 with or without bevacizumab, those in whom all CLMs disappeared on computed tomography were identified. Recurrence of such disappearing lesions in situ was examined on a tumor-by-tumor basis. Five (4\%) patients with a total of 44 CLMs met the evaluation criteria. The median number of CLMs prior to chemotherapy was 8 (range, 2-16). The median maximal diameter of the CLMs was $1.8 \mathrm{~cm}$ (range, 1.0-2.4). The median time-todisappearance of all eligible lesions was 6.5 months (range, 4.5-7.5). Histological examination of scar lesions on the liver surface revealed no viable cancer cells. Two lesions were surgically resected. During clinical follow-up of the remaining 42 lesions, in situ recurrence was observed in 8 . The cumulative 1-, 2- and 3-year rates of relapse in situ were 9.1, 9.1 and $31.1 \%$, respectively. Given the low risk of recurrence in situ, the results suggest that the sites of disappearing CLMs may be left unresected but should be carefully monitored during follow-up, with resection an option if the lesion should recur. However, to validate such a treatment strategy, further investigation with a larger series of patients is warranted.
\end{abstract}

\section{Introduction}

Recent advances in chemotherapy have resulted in an increasing number of patients with colorectal cancer liver metastases (CLMs) being treated with systemic chemotherapy prior to hepatic metastasectomy, either as neoadjuvant treatment for

Correspondence to: Dr Kensuke Kumamoto, Department of Digestive Tract and General Surgery, Saitama Medical Center, Saitama Medical University, 1981 Kamoda, Kawagoe, Saitama 350-8550, Japan

E-mail: kumamotk@saitama-med.ac.jp

Key words: colorectal cancer, liver metastases, oxaliplatin initially resectable lesions or in an attempt to make unresectable lesions resectable. When used as first-line chemotherapy for CLMs, new and effective regimens, including 5-fluorouracil (5-FU)/leucovorin (LV), irinotecan and oxaliplatin in combination with targeted agents, have yielded a complete response in 1 to $9 \%$ of patients with CLMs (1-3). The optimal treatment strategy in such cases, however, remains to be determined as, to the best of our knowledge, little research has been carried out on this topic and the results thus far have been conflicting.

\section{Patients and methods}

Patients. The study protocol conformed to the standards of good practice and ethics of our institution. Informed consent was obtained from the individuals included in the study. A retrospective review of all consecutive patients who had been diagnosed with CLM and who were treated with first-line oxaliplatin-based chemotherapy (modified FOLFOX6; mFOLFOX6) with or without bevacizumab between January 2006 and December 2010 was carried out. The mFOLFOX6 regimen comprised intravenous infusion of oxaliplatin $\left(80 \mathrm{mg} / \mathrm{m}^{2}\right)$ over $2 \mathrm{~h}$, followed by rapid intravenous bolus infusion of 5-FU $\left(400 \mathrm{mg} / \mathrm{m}^{2}\right)$ for $5 \mathrm{~min}$ and continuous intravenous infusion of 5 -FU $\left(2,400 \mathrm{mg} / \mathrm{m}^{2}\right)$ over $46 \mathrm{~h}$. This regimen was repeated every 2 weeks. When used in combination with the mFOLFOX6 regimen, bevacizumab $(5 \mathrm{mg} / \mathrm{kg}$ ) was infused intravenously over $60-90 \mathrm{~min}$ prior to the administration of oxaliplatin. In Japan, the use of oxaliplatin and bevacizumab for metastatic colonic cancer was approved by the governmental health insurance system in March 2005 and April 2007, respectively. During this period, mFOLFOX6 with or without bevacizumab was the standard first-line chemotherapy for metastatic colorectal cancer at our institute.

Data were collected on patients in whom all CLMs initially detected by computed tomography (CT) disappeared during first-line chemotherapy, focusing on time-to-disappearance and time-to-recurrence on a tumor-by-tumor basis.

The clinicopathological patient data recorded included age, gender, site of primary lesion, disease stage at diagnosis of primary lesion, site and number of liver metastases and carcinoembryonic antigen (CEA) level prior to chemotherapy. Adverse events during chemotherapy were evaluated according to the Common Toxicity Criteria of Adverse Events (CTCAE) ver. 4.0 (4). The relative dose intensity of oxaliplatin was also evaluated. 
Table I. Clinical features and details of treatment for each patient.

\begin{tabular}{|c|c|c|c|c|c|c|c|c|c|c|c|}
\hline Case & $\begin{array}{c}\text { Age } \\
\text { (years) }\end{array}$ & Gender & $\begin{array}{l}\text { Stage at } \\
\text { initial } \\
\text { diagnosis }\end{array}$ & $\begin{array}{l}\text { Site of } \\
\text { primary } \\
\text { lesion }\end{array}$ & $\begin{array}{l}\text { CEA at initial } \\
\text { diagnosis } \\
(\mathrm{ng} / \mathrm{ml})\end{array}$ & $\begin{array}{l}\text { No. CLMs } \\
\text { before } \\
\text { mF6 }\end{array}$ & $\begin{array}{c}\text { Maximal } \\
\text { diameter of } \\
\text { tumor }(\mathrm{cm})\end{array}$ & $\begin{array}{l}\text { No. } \\
\text { mF6 } \\
\text { cycles }\end{array}$ & Bev & $\begin{array}{c}\text { Additional mF6+ } \\
\text { Bev after } \\
\text { disappearance }\end{array}$ & $\begin{array}{l}\text { Recurrence } \\
\text { in situ }\end{array}$ \\
\hline 1 & 69 & M & IV & Rectum & 6.6 & 16 & 2.0 & 15 & - & + & + \\
\hline 2 & 35 & $\mathrm{~F}$ & IV & Colon & 14.3 & 8 & 1.8 & 12 & + & - & - \\
\hline 3 & 60 & $\mathrm{~F}$ & II & Colon & 4.5 & 2 & 1.0 & 11 & + & - & + \\
\hline 4 & 68 & M & I & Rectum & 2.0 & 5 & 2.4 & 13 & + & - & + \\
\hline 5 & 68 & $\mathrm{~F}$ & IV & Colon & 5.1 & 13 & 1.4 & 9 & + & + & - \\
\hline
\end{tabular}

mF6, modified FOLFOX6; Bev, bevacizumab. M, male; F, female; CEA, carcinoembryonic antigen; CLMs, colorectal cancer liver metastases .

The extent of metastasis was determined during the pretreatment workup, which usually involved enhanced triple-phase helical CT of the chest, abdomen and pelvis in 5-mm thick slices. CT was periodically performed at 3-4 month intervals. Disappearance was defined as no further lesion or abnormality, including a low attenuated mass, calcification and ring enhancement, at the site of a previously identified CLM. Other imaging modalities, including intravenously enhanced magnetic resonance imaging (MRI) and positron emission tomography (PET)/CT, were also used whenever CT proved inadequate or in order to confirm disappearance on CT.

Statistical analysis. Continuous variables were expressed as the median and range. Time-to-disappearance and time-to-recurrence were estimated on a tumor-by-tumor basis. Time-to-disappearance was defined as the time from the initiation of chemotherapy to radiographic diagnosis of disappearance. Time-to-recurrence was defined as the time from disappearance to the time of initial radiographic evidence of relapse in situ. To calculate the in situ time-to-recurrence of disappearing CLMs, the CLMs were censored at the time of the last image in which no evidence of recurrence was visible. Biopsied lesions without evidence of viable tumor cells were also censored at the time of surgery. The cumulative rates of disappearance and recurrence were estimated using the Kaplan-Meier method.

\section{Results}

Patient characteristics and clinical course. A total of 125 patients diagnosed with CLMs were treated with mFOLFOX6 with or without bevacizumab. In 5 of the patients (4\%), all CLMs disappeared during chemotherapy. Three of the patients were female. The primary site was the colon in 3 patients and the rectum in 2 . At diagnosis of the primary lesion, pathological stage was I in 1 patient, II in 1 and IV in 3. Histological examination revealed well- or moderately differentiated adenocarcinoma in 4 patients and poorly differentiated adenocarcinoma in 1 . The median CEA level (cut-off, $6.7 \mathrm{ng} / \mathrm{ml}$ ) prior to chemotherapy was $5.1 \mathrm{ng} / \mathrm{ml}$ (range, 2.0-14.3). The median number of liver metastases was 8 (range, 2-16). The median maximal diameter of liver metastases per patient was $1.8 \mathrm{~cm}$ (range, 1.0-2.4). The median number of cycles of oxaliplatin-based chemotherapy to disappearance of all CLMs per patient was 12 (range, 9-15), with a median relative dose intensity of oxaliplatin at 79\% (range, 78-88). All patients required a prolonged chemotherapy interval and/or dose reduction due to neutropenia. No peripheral neurotoxicities $>$ grade 3 were observed.

The details of treatment for each patient are summarized in Table I. In Patient 1, CT revealed a large rectal cancer occupying the pelvic space and 16 bilobular metastatic lesions. After 5 and 11 cycles of mFOLFOX6, 12 and 3 CLMs disappeared, respectively. After 15 cycles, the one remaining lesion also disappeared and the primary lesion showed a marked reduction in size. Low anterior resection and biopsy of a scar lesion on the liver surface were performed. Histological examination revealed viable well-differentiated adenocarcinoma cells in the primary lesion but no viable tumor cells in the biopsy specimen. The patient received an additional 6 cycles of mFOLFOX6 postoperatively. At 8 and 9 months after surgery, in situ relapse was detected in 1 and 3 lesions, respectively, on CT and MRI. The patient was administered mFOLFOX6 plus bevacizumab for these 4 lesions, resulting in disappearance of all lesions after 7 cycles. Four months later, one of the 4 lesions reappeared and was subsequently resected. At the second laparotomy, a scar lesion was also resected, revealing no viable tumor cells by histological examination. The patient remains free of disease at 54 months after the initiation of first-line chemotherapy.

In patient 2, CT revealed 8 bilobular synchronous liver metastases from moderately differentiated adenocarcinoma of the transverse colon associated with familial adenomatous polyposis. Chemotherapy comprised mFOLFOX6 plus bevacizumab. After 3 and 12 cycles of mFOLFOX6 plus bevacizumab, 6 and 2 lesions disappeared, respectively. Two months later, the patient underwent total colectomy and biopsy of a scar lesion on the liver surface. Histological examination revealed moderately differentiated adenocarcinoma in the primary tumor but no viable cells in the biopsy specimen. No chemotherapy was administered postoperatively. The patient remains free of disease at 40 months after the initiation of chemotherapy.

Patient 3 received mFOLFOX6 plus bevacizumab therapy for 2 recurrent liver metastases detected at 13 months after Hartmann's procedure for perforated stage II sigmoid colon well-differentiated adenocarcinoma. No hepatectomy was performed due to patient refusal. After 12 cycles of chemotherapy, the 2 metastases disappeared, but reappeared 2 months 


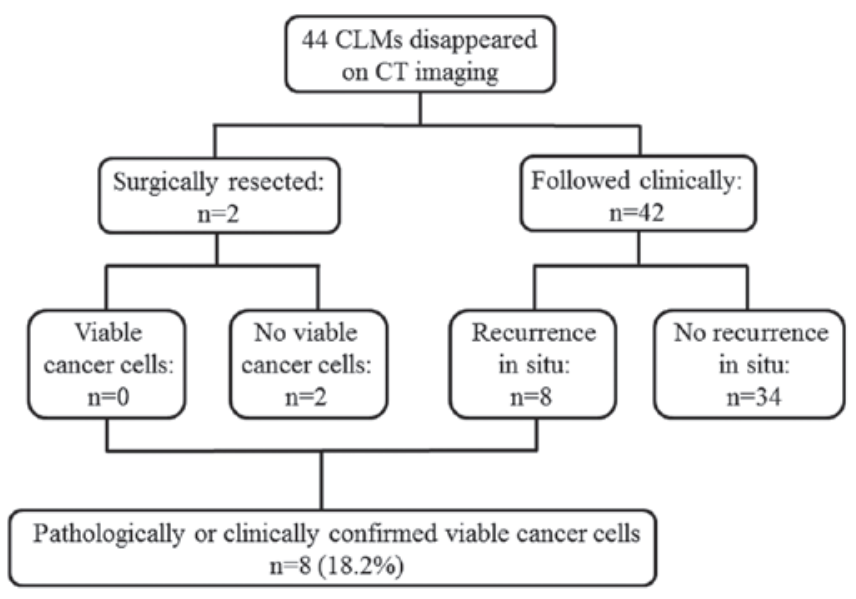

Figure 1. Flow chart of outcome in disappearing CLMs. CLMs, colorectal cancer liver metastases; CT, computed tomography.

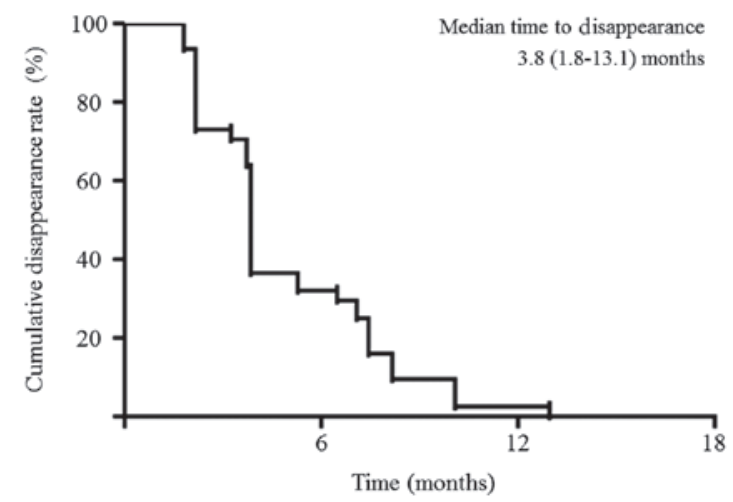

Figure 2. Cumulative disappearance rate of eligible CLMs by Kaplan-Meier method. CLMs, colorectal cancer liver metastases.

later. Subsequent additional chemotherapy included irinotecan plus 5-FU/LV (FOLFIRI) plus bevacizumab and thereafter irinotecan plus cetuximab. However, the patient succumbed to progressive disease at 24 months after the initiation of first-line chemotherapy.

Patient 4 received mFOLFOX6 plus bevacizumab for 5 recurrent bilobular liver metastases at 6 months after abdomino-perineal resection for stage I poorly differentiated adenocarcinoma of the lower rectum. After 12 and 13 cycles of chemotherapy, 2 and 3 lesions disappeared on CT and/or $\mathrm{PET} / \mathrm{CT}$, respectively. Lymph node metastasis along the right internal iliac artery was suspected after 13 cycles. Therefore, the patient was started on FOLFIRI plus bevacizumab. Two metastatic lesions reappeared during chemotherapy. The patient succumbed to progressive disease at 17 months after the initiation of first-line chemotherapy.

Patient 5 received mFOLFOX6 plus bevacizumab for 13 synchonous liver metastases and paraaortic lymph node metastasis at 1 month after resection of moderately differentiated adenocarcinoma of the ascending colon. After 4 and 6 cycles of chemotherapy, 9 and 3 lesions disappeared, respectively. After 9 cycles, the one remaining lesion disappeared and a marked reduction was also observed in the size of the lymph node metastasis. An additional 6 cycles of the same regimen were then administered. Lymph node metastasis was detected

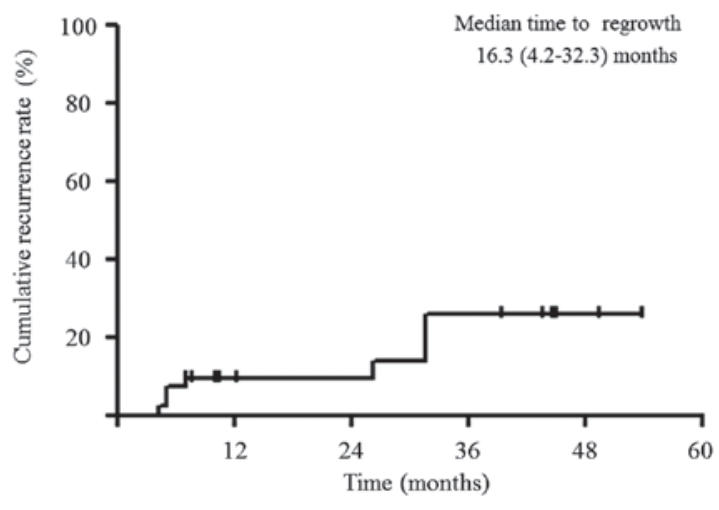

Figure 3. Cumulative recurrence rate of in situ disappearing CLMs by Kaplan-Meier method. CLMs, colorectal cancer liver metastases.

in the hepatoduodenal ligament 3 months later. Percutaneous transhepatic drainage for obstructive jaundice due to hepatic lymph node metastasis was successful, but the patient refused additional chemotherapy. The patient succumbed to disease at 26 months after initiation of first-line chemotherapy.

Time-to-disappearance and time-to-recurrence in situ. Of the 44 lesions evaluated, 2 were resected, revealing no viable tumor cells by histological examination. Of the 42 lesions followed clinically with a median follow-up period of 35.4 months (range, 10.5-58.3), 8 recurred in situ and the remaining 34 did not recur according to radiological evidence. The crude in situ recurrence rate was $18 \%(8 / 44)$, and the true complete response rate, meaning either no viable tumor cells on histological examination or durable local remission of an unresected site, was $80.5 \%$ (36/44; Fig. 1). The median time-to-disappearance was 3.8 months $(1.8-13.1 ;$ Fig. 2). The cumulative 1-, 2- and 3-year rates of recurrence in situ were $9.1,9.1$ and $31.1 \%$, respectively (Fig. 3).

\section{Discussion}

The optimal treatment strategy for CLMs that have disappeared due to new and effective chemotherapy regimens remains to be determined, and a number of problems must be addressed in deciding what the strategy should be. The number of CLMs which disappear or show a reduction in size is not important if they are initially included in the extent of resection. However, when CLMs involve the entire liver, it becomes necessary to consider how the lesions should be dealt with when they disappear without apparent trace. In such cases, a complete cure may be jeopardized if lesions recur due to incomplete eradication of cancerous cells. In fact, no data are available on outcome in patients in whom all sites of CLMs disappearing in situ were left unresected.

In the present study, the true complete response rate was $18 \%$ of disappearing CLMs. The crude recurrence rate in situ may be influenced by the length of the follow-up period, making it difficult to compare between studies. Therefore, we calculated the cumulative rate of in situ recurrence and demonstrated that the 1-, 2- and 3-year rates were 9.1, 9.1 and 31.1\%, respectively.

To the best of our knowledge, including the present study, only 7 studies (5-10) have evaluated the outcome in 
Table II. Studies that evaluated disappearing CLMs on a tumor-by-tumor basis.

\begin{tabular}{lcrr}
\hline Author (ref.) & $\begin{array}{c}\text { Residual cancer in } \\
\text { resected specimen }(\%)\end{array}$ & $\begin{array}{c}\text { Regrowth of clinically } \\
\text { followed lesion (\%) }\end{array}$ & $\begin{array}{c}\text { Residual cancer in } \\
\text { disappeared CLMs (\%) }\end{array}$ \\
\hline Benoist (5) & $12 / 15(80.0)$ & $23 / 31(74.2)$ & $55 / 66(83.3)$ \\
Fiorentini (6) & Not shown & Not shown & $86 / 106(81.1)$ \\
Tanaka (10) & $11 / 45(24.4)$ & $11 / 27(40.7)$ & $22 / 72(30.6)$ \\
Auer (8) & $24 / 68(35.3)$ & $19 / 50(38.0)$ & $43 / 118(36.4)$ \\
van Vledder (9) & $41 / 67(61.2)$ & $21 / 45(46.7)$ & $62 / 112(55.4)$ \\
Present study & $0 / 2(0.0)$ & $8 / 42(19.0)$ & $8 / 44(18.2)$ \\
\hline
\end{tabular}

CLMs, colorectal cancer liver metastases.

disappearing CLMs following chemotherapy. In 3 of these studies, patients were treated with either systemic or hepatic arterial chemotherapy, or both. One study (6) evaluated patients treated with hepatic arterial chemotherapy only. The molecularly-targeted agent bevacizumab or cetuximab were used in combination with systemic chemotherapy in 3 studies, including the present study, with a variety of incidence, ranging from 7.7 to $80 \%(6,9)$. In the present study oxaliplatinbased chemotherapy (mFOLFOX6) was used in all 5 patients and in combination with bevacizumab in 4 patients, since the use of mFOLFOX6 plus bevacizumab was one of the standard therapies for metastatic colorectal cancer during the study period in Japan. Bevacizumab is also known to improve oxaliplatin-related hepatic injuries, including sinusoidal dilatation, sinusoidal obstruction and fibrosis (11), and is thus considered to be suitable for candidates for hepatectomy after oxaliplatinbased chemotherapy.

The details of the 5 studies that evaluated disappearing CLMs on a tumor-by-tumor basis, including our study, are summarized in Table II. Benoist et al (5) examined data on 38 hepatectomized patients with a total of 66 CLMs that disappeared after neoadjuvant systemic chemotherapy with various regimens and reported that persistent macroscopic or microscopic residual disease or early recurrence in situ were observed in 55 lesions (83\%). When the analysis was restricted to lesions left in place at surgery, 23 (74\%) of 31 CLMs were found to have recurred in situ. Fiorentini et al examined 48 patients with a total of 106 CLMs that disappeared following 5-FU-based intra-arterial chemotherapy and reported persistent macroscopic or microscopic evidence of residual disease or early recurrence in situ in 86 lesions (81\%) (6). Auer et al (8) examined data on 39 hepatectomized patients with 118 disappearing CLMs following neoadjuvant chemotherapy comprising various regimens. In their study, 75 of 118 disappearing lesions (64\%), the sites of which were left unresected in subsequent surgery, were considered true complete responses, including 44 pathological complete responses and 31 durable clinical complete responses. A total of 19 disappearing CLMs (38\%) recurred in situ. Tanaka et al (10) reported microscopic evidence of persistent metastases or recurrence in situ in 22 (31\%) of 72 CLMs no longer radiographically visible after neoadjuvant chemotherapy, with $11(41 \%)$ of 27 subsequently unresected lesions recurring in situ. In another study, van Vledder et al (9) analyzed data on 17 hepatectomized patients with disappearing CLMs who were treated with modern anticancer drugs such as oxaliplatin or irinotecan, among whom $91.1 \%$ received concomitant bevacizumab and $41.1 \%$ cetuximab. Of the 45 disappearing CLMs that were unresected, 21 (46.7\%) recurred in situ during a median follow-up period of 20 months. The crude rate of recurrence in situ in our study (18\%) appears to be lower than that reported in earlier studies, which ranges from 38 to $74 \%$. In terms of the cumulative rate of recurrence in situ, the KaplanMeier curve in our study appeared identical to or slightly more favorable than that reported in two previous studies $(8,9)$

CT appears to be the most commonly used imaging modality in the evaluation of the effect of chemotherapy according to RECIST criteria (12). It has been reported that the sensitivity of helical CT is $66-84 \%$ (13-16). In patients with persistent macroscopic disease at surgery, morphological changes in the structure of the liver due to chemotherapy, including steatosis, sinusoidal dilatation and fibrosis, may be responsible for underestimation of liver metastases (17). This raises the question of whether other imaging modalities, such as MRI with liver-specific contrast agents or PET/CT, should be used in patients in whom CLMs are no longer visible on helical CT. Previous studies evaluating the outcome of disappearing CLMs used enhanced CT routinely in combination with ultrasonography $(8,9)$, contrast-enhanced MRI $(10,12)$ or PET/CT (12). In our study, despite a lack of sufficient data on the usefulness of these alternative diagnostic modalities, either enhanced MRI or PET/CT was additionally performed to confirm judgment of the disappearance of lesions on CT imaging.

The present study had a number of limitations, including its retrospective nature and small patient sample. However, the results suggest that outcome in disappearing CLMs during oxaliplatin-based chemotherapy is more favorable than previously reported. Although the precise reason for this improvement remains unclear, one possible explanation is that 4 of the 5 patients were administered mFOLFOX6 plus bevacizumab and that 3 of the 5 patients received additional chemotherapy. It should be noted that there are no supporting data from earlier studies for this supposition. The present data do suggest, however, that studies are warranted on a larger series of patients with disappearing CLMs treated with new anticancer drugs and molecularly-targeted agents.

In terms of the treatment strategy or approach to disappearing CLMs, owing to the high rate of in situ recurrence, 
Benoist et al (5) noted that i) a complete response on imaging did not mean cure in most patients; ii) medical oncologists should refer patients with resectable CLMs to surgeons before any lesions have completely disappeared; and iii) the sites of lesions disappearing with chemotherapy should be resected. Elias et al (7) and Auer et al (8) reported a satisfactory rate of in situ recurrence with hepatic arterial chemotherapy, indicating a satisfactory level of efficacy. However, given the range of new and effective chemotherapy regimens now available worldwide, this approach should be reconsidered given the concomitant technical problems associated with placement and maintenance of the catheter system. van Vleddler et al (9) proposed that aggressive surgery should be considered in patients showing a marked response to chemotherapy, even when all CLM sites could not be identified.

Despite the favorable results observed in the present study, we believe that it is prudent to resect all initially detected sites of CLMs whenever possible. Taking the results of earlier studies into consideration, the following strategies may be appropriate: i) if all the lesions are initially resectable and chemotherapy is administered in an adjuvant setting, then the duration of chemotherapy should be limited; and ii) where preoperative chemotherapy is administered to make initially unresectable lesions resectable, careful follow-up imaging is important to ensure that they are not reduced in size to the point where identifying them intraoperatively would be difficult or impossible for the surgeon. However, the low rate of in situ recurrence of approximately $30 \%$ at 3 years in our study suggests that the sites of disappearing CLMs may be left untouched, only resecting should they recur.

In conclusion, given the low risk of recurrence in situ, the results of the present study suggest that the sites of disappearing CLMs may be left unresected but should be carefully monitored during follow-up, with resection an option if the lesion should recur. These results provide important data on the treatment of disappearing CLMs in the era of new and effective chemotherapy. However, to validate such a treatment strategy, further investigation with larger series of patients is warranted.

\section{Acknowledgements}

The authors would like to thank Associate Professor Jeremy Williams, Tokyo Dental College, for his assistance with the English of the manuscript.

\section{References}

1. Nordlinger B, Sorbye H, Glimelius B, et al; EORTC Gastro-Intestinal Tract Cancer Group; Cancer Research UK; Arbeitsgruppe Lebermetastasen und-tumoren in der Chirurgischen Arbeitsgemeinschaft Onkologie (ALM-CAO); Australasian Gastro-Intestinal Trials Group (AGITG); Fédération Francophone de Cancérologie Digestive (FFCD): Perioperative chemotherapy with FOLFOX4 and surgery versus surgery alone for resectable liver metastases from colorectal cancer (EORTC Intergroup trial 40983): a randomised controlled trial. Lancet 371: 1007-1016, 2008
2. Wong R, Cunningham D, Barbachano Y, et al: A multicentre study of capecitabine, oxaliplatin plus bevacizumab as perioperative treatment of patients with poor-risk colorectal liver-only metastases not selected for upfront resection. Ann Oncol 22: 2042-2048, 2011.

3. Bokemeyer C, Bondarenko I, Makhson A, et al: Fluorouracil, leucovorin, and oxaliplatin with and without cetuximab in the first-line treatment of metastatic colorectal cancer. J Clin Oncol 27: 663-671, 2000.

4. National Cancer Institute: Common terminology criteria for adverse events (CTCAE) v4.0. http://ctep.cancer.gov/ protocolDevelopment/electronic_applications/ctc.htm\#ctc_40. Accessed April 23, 2012.

5. Benoist S, Brouquet A, Penna C, et al: Complete response of colorectal liver metastases after chemotherapy: does it mean cure? J Clin Oncol 24: 3939-3945, 2006.

6. Fiorentini G, Del Conte A, De Simone M, et al: Complete response of colorectal liver metastases after intra-arterial chemotherapy. Tumori 94: 489-492, 2008.

7. Elias D, Goere D, Boige V, Kohneh-Sharhi N, Malka D, Tomasic G, Dromain C and Ducreux M: Outcome of posthepatectomy-missing colorectal liver metastases after complete response to chemotherapy: impact of adjuvant intra-arterial hepatic oxaliplatin. Ann Surg Oncol 14: 3188-3194, 2007.

8. Auer RC, White RR, Kemeny NE, et al: Predictors of a true complete response among disappearing liver metastases from colorectal cancer after chemotherapy. Cancer 116: 1502-1509, 2010.

9. van Vledder MG, de Jong MC, Pawlik TM, Schulick RD, Diaz LA and Choti MA: Disappearing colorectal liver metastases after chemotherapy: should we be concerned? J Gastrointest Surg 14: 1691-1700, 2010.

10. Tanaka K, Takakura H, Takeda K, Matuo K, Nagano Y and Endo I: Importance of complete pathologic response to prehepatectomy chemotherapy in treating colorectal cancer metastases. Ann Surg 250: 935-942, 2009.

11. Klinger M, Epieldauer S, Hacker S, et al: Bevacizumab protects against sinusoidal obstruction syndrome and does not increase response rate in neoadjuvant XELOX/FOLFOX therapy of colorectal cancer liver metastases. Eur J Surg Oncol 35: 515-520, 2009.

12. Eisenhauer EA, Therasse P, Bogaerts J, et al: New response evaluation criteria in solid tumors: revised RECIST guideline (version 1.1). Eur J Cancer 45: 228-247, 2009.

13. Bhattacharjya S, Bhattacharjya T, Baber S, Tibballs JM, Watkinson AF and Davidson BR: Prospective study of contrastenhanced computed tomography, computed tomography during arterioportography, and magnetic resonance imaging for staging colorectal liver metastases for liver resection. Br J Surg 91: 1361-1369, 2004.

14. Bipat S, van Leeuwen MS, Comans EF, et al: Colorectal liver metastases: CT, MR imaging, and PET for diagnosis - metaanalysis. Radiology 237: 123-131, 2005.

15. Vallas C, Andía E, Sánchez A, et al: Hepatic metastases from colorectal cancer: Preoperative detection and assessment of respectability with helical CT. Radiology 218: 55-60, 2001.

16. Elias D, Sideris L, Pocard M, et al: Incidence of unsuspected and treatable metastatic disease associated with operable colorectal liver metastases discovered only at laparotomy (and not treated when performing percutaneous radiofrequency ablation). Ann Surg Oncol 12: 298-302, 2005.

17. Fernandez FG, Ritter J, Goodwin JW, Linehan DC, Hawkins WG and Strasberg SM: Effect of steatohepatitis associated with irinotecan or oxaliplatin pretreatment on resectability of hepatic colorectal metastases. J Am Coll Surg 200: 845-853, 2005. 